\title{
Efeitos da caminhada nórdica
} no perfil antropométrico e composição corporal de pessoas com doença de Parkinson: ensaio clínico randomizado

\author{
Effect of Nordic Walking on anthropometric profile and body composition \\ of subjects with Parkinson's disease: randomized clinical trial
}

\author{
Priscila Antunes Marques \\ Elren Passos Monteiro² \\ Alex de Oliveira Fagundes ${ }^{3}$ \\ Rochelle Rocha da Costa ${ }^{4}$ \\ Flávia Gomes Martinez

\section{Aline de Souza Pagnussat ${ }^{6}$} \\ Leonardo Alexandre Peyré-Tartaruga? \\ Endereço para Correspondência: \\ Leonardo A. Peyré-Tartaruga \\ Laboratório de Pesquisa do Exercício - UFRGS \\ Rua Felizardo, 750 \\ 90690-200 - Porto Alegre, RS [Brasil] \\ leonardo.tartaruga@ufrgs.br
}

1 Programa de Pós Graduação em Ciências do Movimento Humano. Pontifícia Universidade Católica do Rio Grande do Sul - PUC-RS. Porto Alegre, RS - Brasil.

ORCID: https://orcid.org/0000-0001-5060-4142

2 Programa de Pós Graduação em Ciências da Saúde.

Universidade Federal de Ciências da Saúde de Porto Alegre UFCSPA. Porto Alegre, RS - Brasil.

ORCID: https://orcid.org/0000-0001-7757-6620

3 Programa de Pós Graduação em Ciências do Movimento Humano. Universidade Federal do Rio Grande do Sul UFRGS. Porto Alegre, RS - Brasil.

ORCID: https://orcid.org/0000-0002-3246-0613

4 Programa de Pós Graduação em Ciências do Movimento Humano. Universidade Federal do Rio Grande do Sul UFRGS. Porto Alegre, RS - Brasil.

ORCID: https://orcid.org/0000-0003-0182-4936

5 Programa de Pós Graduação em Ciências do Movimento Humano. Universidade Federal do Rio Grande do Sul UFRGS. Porto Alegre, RS - Brasil.

ORCID: https://orcid.org/0000-0001-6838-5080

6 Programa de Pós Graduação em Ciências da Reabilitação, Departamento de Fisioterapia, Laboratório de Análises do Movimento e Reabilitação Neuromuscular. Universidade Federal de Ciências da Saúde de Porto Alegre - UFCSPA. Porto Alegre, RS - Brasil.

ORCID: https://orcid.org/0000-0001-7837-5855

7 Programa de Pós Graduação em Ciências do Movimento Humano. Universidade Federal do Rio Grande do Sul UFRGS. Porto Alegre, RS - Brasil.

ORCID: https://orcid.org/0000-0003-1742-5016

\section{Resumo}

Introdução: A doença de Parkinson (DP) é caracterizada por distúrbios motores, aumentando risco de quedas e inatividade física. Objetivo: Analisar perfil antropométrico e composição corporal, antes e após um programa de treinamento de caminhada nórdica $(\mathrm{CN})$ e caminhada livre (CL). Métodos: Os 33 sujeitos foram randomizados em dois grupos experimentais: CL $(n=16)$ e $C N(n=17)$ para realização das 12 sessões de treinamento. Resultados: Relação cintura/quadril reduziu $(2,2 \%, \mathrm{p}=$ 0,022) para o CL. O somatório total das dobras cutâneas reduziu em 4,3\% no grupo $C N(p=0,012)$, e $4,7 \%$ no grupo $C L(p=0,012)$, sem diferença entre os grupos. $O$ período de treinamento não promoveu alterações significativas na massa corporal $(\mathrm{p}=0,808)$, IMC $(\mathrm{p}=0,392)$, nas massas magra $(\mathrm{p}=0,834)$, gorda $(\mathrm{p}=0,721)$ e óssea $(\mathrm{p}=0,348)$, bem como nos percentuais de gordura $(p=0,887)$. Conclusão: $O$ treinamento promoveu melhora antropométrica efetiva para ambos os grupos.

Descritores: Composição corporal. Metabolismo energético. Treinamento. Doença de Parkinson.

\begin{abstract}
Introduction: Parkinson's disease (DP) is characterized by motor disorders, increasing risk of falls and physical inactivity. Objective: To analyze the anthropometric profile and body composition of subjects before and after participating in a Nordic Walking (NW) or Free Walking (FW) training program. Methods: A total of 33 patients were randomized into two experimental groups, NW $(\mathrm{n}=16)$ and $\mathrm{FW}(\mathrm{n}=17)$, to perform the 12 training sessions. Results: Waist-hip ratio was reduced $(2.2 \%, \mathrm{p}=$ $0.022)$ for FW. The total number of skinfolds was reduced by $4.3 \%$ in the NW group ( $p=0.012)$ and $4.7 \%$ in the FW group $(p=0.012)$. However, the training period did not promote significant changes in body mass $(p=0.808)$, in IMC $(p=0.392)$, lean mass $(p=0.834)$, adipose mass $(p=$ $0.721)$ and bone mass $(p=0.348)$, nor in the percentages of adipose mass $(p=0.887)$ in any of the groups. Conclusion: The training promoted an effective anthropometric improvement for both groups.
\end{abstract}

Keywords: Body composition. Energy metabolism. Training. Parkinson's disease. 


\section{Introdução}

A doença de Parkinson (DP) é uma doença neurológica, crônica, degenerativa e progressiva. Seus sintomas geralmente manifestam-se em torno de 60 anos de idade, sendo homens mais acometidos do que mulheres ${ }^{1}$. No Brasil, o número de pessoas com DP é de aproximadamente 400 mil, e até o ano de 2020 estima-se que mais de quarenta milhões de pessoas terão DP. A projeção para 2040 indica que essa doença seja a segunda causa de morte entre doenças neurológicas em idosos ${ }^{2}$.

As características clínicas da doença são distúrbios motores, tais como tremor em repouso, rigidez, déficits no equilíbrio e na marcha, além de lentidão e redução na amplitude dos movimentos com dificuldade em iniciá-los ${ }^{3}$. Tais alterações podem promover um grande risco de que$\mathrm{das}^{4}$, e associados ao medo de cair, contribuem para a inatividade física, agravando ainda mais o quadro clínico de pessoas com DP e influenciando negativamente na sua composição corporal ${ }^{5,6}$.

A abordagem medicamentosa é o tratamento padrão para a DP, mas o uso do L-dopa apresenta perda da eficácia com o tempo e está associado ao desenvolvimento de complicações motoras típicas, como discinesias ${ }^{4,5}$. Tem sido sugerido que os exercícios físicos podem estimular o controle do movimento e retardar a progressão da doença. Exercícios como a dança ${ }^{7}$ e caminhadas são indicados para este público ${ }^{8}$.

Uma variação da caminhada livre (CL), e possivelmente mais segura para pessoas com DP devido à maior estabilidade através do uso dos bastões, é a caminhada nórdica $(\mathrm{CN})$, do inglês Nordic Walking. Vários estudos já têm demonstrados os efeitos superiores da caminhada nórdica quando comparada a caminhada livre nesses pacientes, em mobilidade funcional, questões cognitivas, ativação muscular ${ }^{9}$. Utilizando-se dos bastões, o comprimento da passada aumenta, resultando em uma marcha mais rápida e segura ${ }^{10}$. Além de proporcionar uma maior ativação muscular na parte superior do corpo durante a caminhada, aumentando o gasto calórico quando comparada à $\mathrm{CL}^{11-13}$. Entretanto, o manejo de terapias físicas exige o monitoramento do perfil nutricional, pois, normalmente, a massa corporal e massa magra são reduzidas em pessoas com doença de Parkinson em comparação com sujeitos controle ${ }^{14,15}$. Inclusive estes prejuízos de composição corporal são indicadores de demência e declínio cognitivo na $\mathrm{DP}^{16}$.

Embora a DP seja muito estudada quanto a diferentes aspectos clínico-funcionais, pouco se sabe em relação aos efeitos da $\mathrm{CN}$ sobre o perfil antropométrico e a composição corporal em pessoas com essa doença. E mais, ao que se sabe, até o presente momento, nenhuma pesquisa mostrou os efeitos de diferentes programas de treinamento de caminhadas (CN e CL) nos parâmetros da composição corporal nesta população. Sendo assim, o objetivo no atual estudo é avaliar os efeitos de um programa de $\mathrm{CN}$ e um de CL sobre o perfil antropométrico e a composição corporal de pessoas com DP, e correlacionar o estadiamento da DP com o índice de massa corporal (IMC) e percentual de gordura dos participantes. Hipotetizou-se que a $\mathrm{CN}$ possa melhorar o quadro de composição corporal (percentual de gordura e massa livre de gordura) em maior grau do que a CL, devido às diferenças biomecânicas entre os tipos de movimento.

\section{Materiais e métodos}

Este estudo caracteriza-se como longitudinal do tipo ensaio clínico randomizado em paralelo, tendo sido registrado no ClinicalTrials. gov (NCT03355521), e conduzido após a aprovação pelo Comitê de Ética do Hospital de Clínicas de Porto Alegre (HCPA) com o número 555.123, e foi descrito seguindo as recomendações do CONSORT (http://www.consort-statement. $\mathrm{org} /$ ). Os procedimentos avaliativos foram realizados no Laboratório de Pesquisa do Exercício (LAPEX) da Universidade Federal do Rio Grande do Sul (UFRGS).

O recrutamento dos voluntários ocorreu durante consultas no ambulatório e análise de 
22 prontuários do setor de neurologia do HCPA e de prontuários de duas Unidades Básicas de Saúde (SUS) da cidade de Porto Alegre. Ainda foi realizada uma divulgação em três jornais de ampla circulação, via redes sociais, e na Associação de Parkinson do Rio Grande do Sul (APARS).

Participaram do estudo homens e mulheres, com idade acima de 50 anos, com DP idiopática, com o diagnóstico atribuído por neurologistas, por meio da utilização dos Critérios do Banco de Cérebro de Londres (CBCL) ${ }^{17}$. Como critérios de elegibilidade: (1) não ter realizado cirurgias recentes, estimulação cerebral profunda (DBS - Deep Brain Stimulation); (2) ter cardiopatias graves, hipertensão arterial não controlada, ou ter sofrido infarto do miocárdio há menos de um ano, ou ser portador de marcapasso; (3) ter sido acometido por acidente cerebral encefálico ou outras doenças neurológicas associadas, ou ter demência; (4) usar próteses nos membros inferiores; (5) não ter condições de deambulação; (6) ter atestado médico com liberação para participar de treinamentos com duração em torno de 60 minutos; (7) estar em tratamento médico e medicamentoso; (8) estar isento da prática de exercícios físicos regulares há pelo menos seis meses.

Os testes foram realizados em uma única visita, efetuando-se avaliações de antropometria, composição corporal e estadiamento da doença. Os participantes receberam assistência de dois pesquisadores para evitar possíveis quedas, durante as avaliações as quais ocorreram na fase "ON" da medicação antiparkinsoniana. Após os procedimentos avaliativos, os indivíduos foram randomizados (via online pelo site www.randomization.org) alocados nos diferentes grupos de intervenção por um avaliador cegado. Os sujeitos foram avaliados antes e após o período de treinamento pelos mesmos avaliadores, cegados quanto ao tipo de intervenção.

A massa corporal foi mensurada em uma balança científica, com capacidade de $200 \mathrm{~kg}$ e resolução de 100 g; e a estatura, em um estadiômetro acoplado à referida balança, com resolu- ção de $1 \mathrm{~mm}$ (Filizola, São Paulo, Brasil). O índice de massa corporal (IMC) foi determinado pela razão entre a massa corporal e a estatura ao quadrado. Na sequência, um avaliador experiente, mensurou perímetros, diâmetros ósseos e comprimento do membro inferior.

As medidas antropométricas foram realizadas seguindo a recomendação da International Standards for Anthropometric Assessment ${ }^{18}$. Esta avaliação ocorreu duas vezes em forma de circuito nas dobras cutâneas: peitoral, axilar média, tricipital, subescapular, suprailíaca, abdominal, coxa e panturrilha mensuradas por um adipômetro científico da marca Sanny (American Medical, São Paulo, Brasil), com resolução de 0,1 mm. Considerou-se uma média das medidas e quando havia uma diferença de $10 \%$, uma terceira mensuração era realizada pelo mesmo avaliador. O percentual de gordura foi obtido por meio da fórmula de Siri ${ }^{19}: \% \mathrm{G}=$ [(4,95/DENSIDADE C.) - 4,50] X 100.

A avaliação dos sintomas motores e o estadiamento da DP foram realizados por um avaliador experiente, por meio das escalas UPDRS, Hoehn \& Yahr (H\&Y), respectivamente. A escala UPDRS - III, validada para a população brasileira ${ }^{20}$, contém 14 itens correspondentes à sessão de exploração motora que contemplam os seguintes domínios: fala, expressão facial, tremor de repouso, tremor postural e de ação das mãos, rigidez, movimentos manuais, agilidade das pernas, levantar da cadeira, postura, marcha, estabilidade postural e bradicinesia e hipocinesias corporais, cujos escores variam de 18 a 31, de forma que quanto maior o escore, maior o comprometimento.

O estadiamento da doença foi avaliado com a escala Hoehn e Yahr (H\&Y), também validada para a população brasileira21. Esta escala possui oito estágios, do 0 (sem sinais da doença) até o 5 (uso de cadeiras de rodas e/ou acamado) de acordo com a gravidade da DP, como podem ser observados no Quadro 1:

Durante seis semanas, os sujeitos compareceram à Escola de Educação Física, Fisioterapia e Dança (EsEFID-UFRGS) para a realização das 


\begin{tabular}{|c|c|}
\hline Estadiamento & Sintomas \\
\hline Estágio 0 & Sem sinais da doença. \\
\hline Estágio 1 & Doença unilateral. \\
\hline Estágio 1,5 & Acometimento unilateral e axial. \\
\hline Estágio 2 & $\begin{array}{c}\text { Acometimento bilateral, } \\
\text { sem prejuízo do equilíbrio }\end{array}$ \\
\hline Estágio 2,5 & $\begin{array}{c}\text { Leve acometimento bilateral, } \\
\text { recuperação no teste de equilíbrio } \\
\text { ("pull test"). }\end{array}$ \\
\hline Estágio 3 & $\begin{array}{c}\text { Acometimento leve a moderado; } \\
\text { alguma instabilidade postural; } \\
\text { independente fisicamente. }\end{array}$ \\
\hline Estágio 4 & $\begin{array}{c}\text { Acometimento severo; ainda capaz } \\
\text { de caminhar ou permanecer em pé } \\
\text { sem auxílio. }\end{array}$ \\
\hline Estágio 5 & $\begin{array}{c}\text { Usando cadeira de rodas ou aca- } \\
\text { mado exceto se auxiliado. }\end{array}$ \\
\hline
\end{tabular}

Quadro 1: Escala Hoehn e Yahr (H\&Y) para o estadiamento e para o nível de incapacidade da Doença de Parkinson Fonte: Scalzo et al. ${ }^{21}$.

12 sessões de treinamento de $\mathrm{CN}$ e de $\mathrm{CL}$, com a frequência de duas vezes por semana, em dias não consecutivos, com a duração inicial de 35 minutos diários, com progressão até 50 minutos no total, no último ciclo de treinamento, totalizando 12 sessões. Os participantes de ambos os grupos fizeram seis sessões de familiarização da caminhada e correção da técnica (Quadro 2). Os mesmos exercícios foram realizados nos dois grupos, porém no grupo da caminhada nórdica foi introduzido o uso do bastão (Newfeel® - Decathlon, Nordic Walker, China) que foi ajustado individualmente conforme a estatura corporal. O protocolo de familiarização e ensino da técnica de $\mathrm{CN}$ foi realizado segundo o estudo de Arcila et al. ${ }^{15}$.

O programa de treinamento tanto da $\mathrm{CN}$ quanto da CL consistiu em três momentos, a saber: mobilização articular + aquecimento com caminhada livre de três minutos na velocidade de caminhada autosselecionada (VAS); parte principal constituída pelo treinamento específico do grupo e volta à calma. Maiores detalhes sobre a periodização do treinamento, ver o estudo de Monteiro ${ }^{5}$.

\section{Anólise estatístico}

O tamanho da amostra foi calculado antes do início do estudo, usando o software G-Power, para Windows, versão 3.1.9.2. Foi adotado um nível de significância de 0,05 , um poder com $1-\beta$ probabilidade de erro de $95 \%$, assumindo um $\alpha$ $=0,05$, tamanho de efeito de 0,43, baseado nas médias e desvios-padrão do percentual de gordura do grupo caminhada nórdica e caminha livre do estudo de Song et al. (2013). O resultado do tamanho amostral foi o de 14 participantes para cada grupo, porém considerando uma possível perda amostral, selecionaram-se 75 voluntários, restando, destes, 33 participantes $(\mathrm{CN}, \mathrm{n}$ = 17) $(\mathrm{CL}, \mathrm{n}=16)$.

Os dados das variáveis escalares do atual estudo estão apresentados em média e erro padrão, enquanto os dados das variáveis categóricas em frequência absoluta (n). A comparação dos dados de caracterização da amostra nos diferentes grupos foi realizada por meio de um

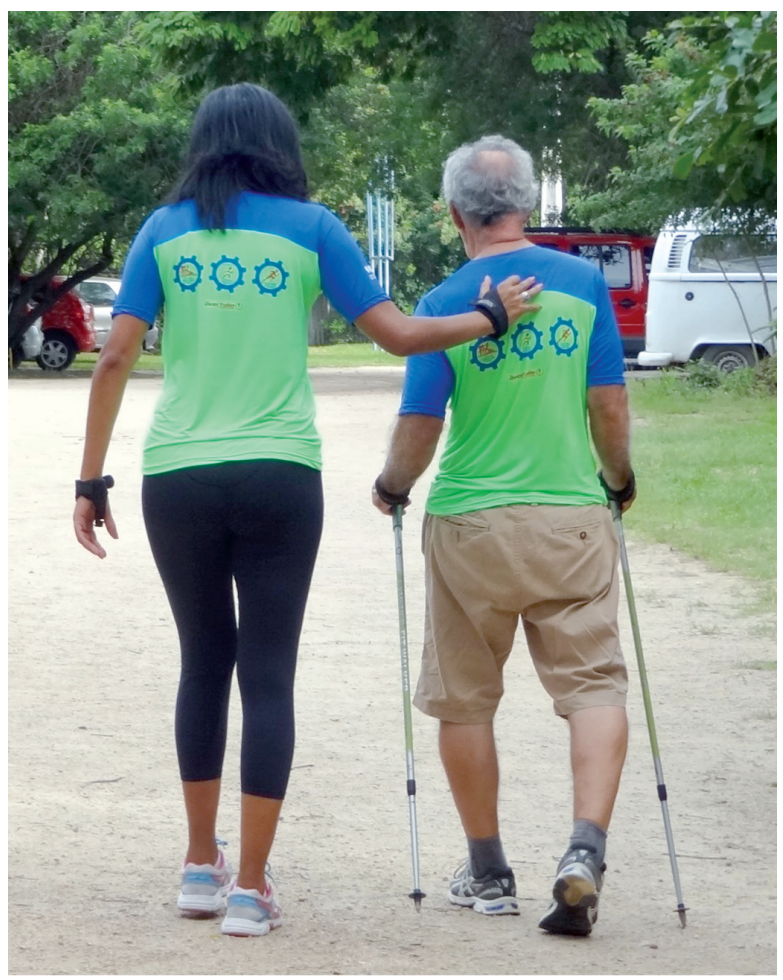

Figura l: Familiarização da técnica da caminhada nórdica

Fonte: Os autores. 


\begin{tabular}{|c|c|}
\hline Sessões & Exercícios da sessão \\
\hline $\begin{array}{l}\text { Sessão 1: } \\
\text { Postura, fortalecimento do } \\
\text { abdômen e equilíbrio }\end{array}$ & $\begin{array}{l}\text { - Contrair rápida e lentamente o abdômen } \\
\text { - Apoiar-se em um pé com olhos abertos e fechados } \\
\text { - Estender e flexionar joelhos (estático) } \\
\text { - Projetar o peitoral para frente } \\
\text { - Manter a postura ereta } \\
\text { - Colocar bastões e arrastrar }\end{array}$ \\
\hline $\begin{array}{l}\text { Sessão 2: } \\
\text { Correção dos padrões da } \\
\text { marcha }\end{array}$ & $\begin{array}{l}\text { - Colocar os pés em diferentes ângulos } \\
\text { - Caminhar em diferentes direções: para frente, para trás, para o lado e na diagonal } \\
\text { - Balançar apoiando-se ora no calcanhar, ora na ponta dos pés } \\
\text { - Colocar primeiro o calcanhar no chão ao pisar } \\
\text { - Impulsar o corpo à frente apoiando-se na ponta do pé } \\
\text { - Estender os joelhos para impulsar o corpo } \\
\text { - Flexionar os joelhos para dar passo à frente } \\
\text { - Caminhar alternando braço direito com perna esquerda e vice-versa. }\end{array}$ \\
\hline $\begin{array}{l}\text { Sessão 3: } \\
\text { Dissociação das cinturas } \\
\text { pélvica e escapular }\end{array}$ & $\begin{array}{l}\text { - Balançar os braços e rotacionar o tronco com e sem bastões (estático) } \\
\text { - Caminhar com e sem bastões focando no balanço de braços e na rotação de tronco } \\
\text { - Posicionar-se em dupla, um atrás do outro: uma pessoa segura o bastão pelo agarre, } \\
\text { e a outra pela ponta, deve-se rotar o tronco e balançar os braços simultaneamente }\end{array}$ \\
\hline $\begin{array}{l}\text { Sessão 4: } \\
\text { Coordenação entre braços } \\
\text { e pernas }\end{array}$ & $\begin{array}{l}\text { - Caminhar com os braços em movimentos simultâneos para frente e para trás, } \\
\text { pressionando o bastão no chão a fim de impulsar o corpo à frente } \\
\text { - Realizar o mesmo exercício anterior focando na semiflexão de cotovelo } \\
\text { - Caminhar com o auxílio de bastões procurando pressioná-los no chão, alternando o } \\
\text { braço direito com perna esquerda e vice-versa } \\
\text { - Caminhar com o apoio de bastões, estender o cotovelo e inclinar os bastões } \\
\text { para trás } \\
\text { - Formar dupla, um atrás do outro: uma pessoa segura o bastão pelo agarre, e a outra } \\
\text { pela ponta e caminhar movimentando os bastões }\end{array}$ \\
\hline $\begin{array}{l}\text { Sessão 5: } \\
\text { Trabalhar a amplitude de } \\
\text { movimento e aumentar a } \\
\text { velocidade da marcha }\end{array}$ & $\begin{array}{l}\text { - Caminhar com os bastões com a atenção no comprimento do passo (passo longo) } \\
\text { - Caminhar com os bastões e abrir as mãos quando os bastões forem movimentados } \\
\text { para trás, e fechá-las quando estes forem impulsionados para frente }\end{array}$ \\
\hline $\begin{array}{l}\text { Sessão 6: } \\
\text { Técnica completa }\end{array}$ & $\begin{array}{l}\text { - Caminhar tendo em conta todos os aspectos técnicos da marcha: } \\
\text { - Dar passo longo } \\
\text { - Fazer contato inicial do calcanhar no chão ao caminhar } \\
\text { - Alongar verticalmente, manter a postura ereta, olhar para o horizonte } \\
\text { - Coordenar braços e pernas e na caminhada nórdica: } \\
\text { - Realizar semiflexão de cotovelo } \\
\text { - Inclinar os bastões para trás } \\
\text { - Fazer descarga de peso nos bastões para impulsar o corpo à frente } \\
\text { - Abrir e fechar as mãos }\end{array}$ \\
\hline
\end{tabular}

Quadro 2: Protocolo de familiarização para o ensino da técnica da CN e correção da caminhada

Fonte: Adaptado de Arcila et al. ${ }^{15}$.

teste $t$ para amostras independentes, a normalidade pelo teste de Shapiro-Wilk e pelo teste Qui-Quadrado. Para a análise das variáveis dependentes, na comparação dos diferentes grupos nos momentos pré- e pós-treinamento, utilizouse as Equações de Estimativas Generalizadas (GEE) com post hoc de Bonferroni. Além disso, o tamanho de efeito foi calculado pelo método "d" de Cohen, comparando as médias pré- e pósdentro de cada grupo. O teste de Spearman foi usado para a análise da correlação entre estadiamento da doença e composição corporal. As análises foram realizadas no software SPSS, para Mac, versão 22.0, com $\alpha<0,05$. 


\section{Resultados}

Embora a amostra inicial deste estudo tenha se constituído de 33 sujeitos divididos randomicamente entre os grupos CL $(n=16)$ e $C N$ ( $\mathrm{n}=17)$, quatro participantes não concluíram o período de seis semanas de intervenções (dois do CL e dois do $\mathrm{CN}$ ), representando $12 \%$ de desistência. Além destes, outros quatro sujeitos não realizaram as avaliações pós-treinamento. Assim, 29 participantes finalizaram as sessões de treinamento físico, e 25 realizaram todas as avaliações propostas neste trabalho (Figura 2). Aqueles que completaram o período de intervenções apresentaram frequência superior a $90 \%$ nas sessões de treinamento, demonstrando aderência ao protocolo proposto. As características dos participantes estão apresentadas na Tabela 1.
Decorridas seis semanas de intervenções, foram observadas mudanças significativas nos desfechos relação cintura/quadril, somatório de dobras cutâneas de membros superiores e somatório de dobras cutâneas. Os valores da relação cintura/quadril foram reduzidos em 2,2\% após o protocolo de CL ( $p=0,022$; tamanho do efeito: $0,24 \pm 0,38)$. O somatório das dobras cutâneas referentes aos MMSS sofreu redução de 5,5\%, depois da CN ( $\mathrm{p}=0,017$; tamanho do efeito: 0,18 $\pm 0,37$ ); e 4,6\%, após CL ( $\mathrm{p}=0,017$; tamanho do efeito: $0,17 \pm 0,38$ ). Além disso, o somatório total das dobras cutâneas apresentou diminuição significativa de $4,3 \%$ no grupo $\mathrm{CN}$ ( $\mathrm{p}=0,012$; tamanho do efeito: $0,14 \pm 0,37$ ); e $4,7 \%$, no grupo CL (p $=0,012$; tamanho do efeito: $0,16 \pm 0,37$ ). Os dados referentes à antropometria são apresentados na tabela 2.

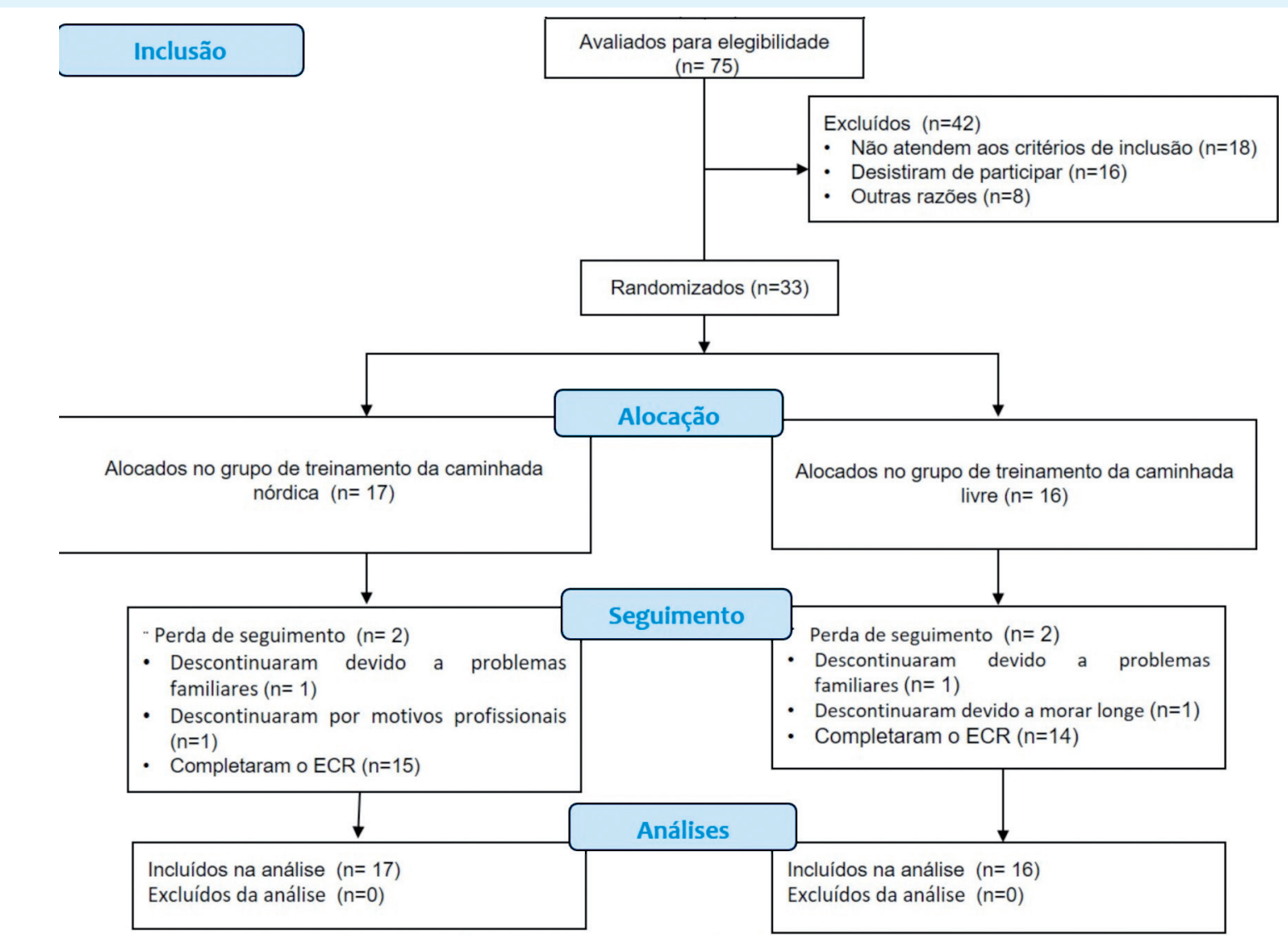

Figura 2: Fluxograma demonstrativo do processo de seleção, alocação e anólise dos participantes do estudo. Doença de Parkinson (DP); estimulação cerebral profunda (DBS Deep Brain Stimulation); ensaio clínico controlado randomizado (ECR)

Fonte: Elaborada pelos autores. 
Tabela 1: Dados de caracterização dos grupos CN e CL, apresentados em média e erro padrão (desfechos escalares) e frequência absoluta (desfechos categóricos), e os índices de significância das comparações entre grupos no pré-teste

\begin{tabular}{lccc}
\hline & $\begin{array}{c}\text { Grupo CN } \\
(\boldsymbol{n}=\mathbf{1 7})\end{array}$ & $\begin{array}{c}\text { Grupo CL } \\
(\boldsymbol{n}=\mathbf{1 6})\end{array}$ & $\boldsymbol{p}$ \\
\hline Idade (anos) & $64,43 \pm 10,14$ & $70,71 \pm 5,95$ & 0,059 \\
Sexo (feminino/masculino) & $6 / 11$ & $6 / 10$ & 0,035 \\
Tempo de diagnóstico da DP (meses) & $60,50 \pm 45,74$ & $58,71 \pm 40,63$ & 0,914 \\
UPDRS III & $15,79 \pm 14,14$ & $23,36 \pm 16,91$ & 0,210 \\
Hoehn e Yahr 1 (n) & 7 & 5 & \\
Hoehn e Yahr 2 $(\mathrm{n})$ & 6 & 6 & 0,217 \\
Hoehn e Yahr 2,5 $(\mathrm{n})$ & 3 & 2 & \\
Hoehn e Yahr 4 $(\mathrm{n})$ & 1 & 3 & \\
\hline
\end{tabular}

Fonte: Elaborada pelos autores. mento da doença e o IMC. Dessa forma, não há influência do grupo. Para realizá-la utilizou-se a amostra total do estudo, assim proporcionando um maior poder de análise. Ao se observarem as correlações entre o estadiamento da DP pela escala H\&Y e o IMC $(\rho=-0,292$ e p $=0,131$; e $\rho=-0,257$ e $\mathrm{p}=0,187)$, e a H\&Y com o percentual da gordura corporal $(\rho=-0,180$ e p $=0,368$; e $\rho=$ $-0,140$ e $\mathrm{p}=0,485)$, não se encontrou nenhuma correlação significativa nos dados da amostra pré- e póstreinamento, respectivamente.
No entanto, o protocolo de treinamento proposto, tanto para $\mathrm{CN}$ quanto para $\mathrm{CL}$, não foi eficaz para mudanças significativas na massa corporal $(p=0,808)$, no IMC $(p=0,392)$, no MMII ( $p=0,309)$, nas massas magra $(p=0,834)$, gorda $(p=0,721)$ e óssea $(p=0,348)$, bem como nos percentuais de gordura $(\mathrm{p}=0,887)$, de massa muscular ( $\mathrm{p}=0,361)$ e de massa óssea ( $\mathrm{p}=0,348)$.

Objetivou-se, na análise da correlação, verificar se existem associações entre o estadia-

\section{Discussão}

O objetivo neste estudo foi analisar o perfil antropométrico e a composição corporal de pessoas com DP, antes e após seis semanas de treinamento de $\mathrm{CN}$ e $\mathrm{CL}$, e verificar a existência de associação entre o estadiamento da DP com o IMC e o percentual de gordura dos participantes. A hipótese aqui proposta não foi confirmada, pois, embora o protocolo de treinamento de

Tabela 2: Desfechos relacionados à antropometria dos pacientes com DP dos grupos CN e CL no pré- e no pós-treinamento. Dados apresentados em média e erro-padrão

\begin{tabular}{lcccc}
\hline & \multicolumn{2}{c}{ Grupo Caminhada Nórdica } & \multicolumn{2}{c}{ Grupo Caminhada Livre } \\
\hline & Pré-Treinamento & Pós-Treinamento & Pré-Treinamento & Pós-Treinamento \\
\hline Massa Corporal (kg) & $81,46(4,20)$ & $82,17(4,42)$ & $66,66(3,03)^{*}$ & $65,68(3,62)^{*}$ \\
IMC (kg/m ${ }^{2}$ ) & $28,53(1,30)$ & $28,65(1,34)$ & $25,92(1,01)$ & $25,49(1,20)$ \\
Relação Cintura/Quadril & $0,94(0,02)$ & $0,95(0,02)$ & $0,91(0,02)$ & $0,89(0,02)^{*} \#$ \\
MMII (mm) & $27,41(2,97)$ & $27,71(2,73)$ & $36,00(4,07)$ & $34,19(4,06)$ \\
MMSS (mm) & $123,87(9,15)$ & $117,00(9,09) \#$ & $103,67(6,93)$ & $98,85(6,74) \#$ \\
Somatório Dobras Cutâneas & $151,28(11,39)$ & $144,71(10,77) \#$ & $139,67(9,94)$ & $133,04(9,69) \#$ \\
Massa Magra (kg) & $63,43(2,88)$ & $64,19(3,01)$ & $51,02(2,14)^{*}$ & $50,87(3,39)^{*}$ \\
Massa Gorda (kg) & $18,02(2,06)$ & $17,99(2,26)$ & $15,64(2,09)$ & $14,81(1,79)$ \\
Massa Óssea (kg) & $16,98(4,03)$ & $17,02(4,03)$ & $14,94(3,75)$ & $14,95(3,75)$ \\
\%Gordura & $21,62(1,64)$ & $21,27(1,84)$ & $22,74(2,41)$ & $22,58(2,52)$ \\
\%Massa Muscular & $37,59(1,70)$ & $37,78(1,97)$ & $38,49(2,54)$ & $35,26(2,10)$ \\
\%Massa Óssea & $20,99(4,84)$ & $20,73(4,59)$ & $22,61(5,69)$ & $23,29(5,99)$ \\
\hline
\end{tabular}

* indica haver diferença significativa entre os grupos no mesmo tempo. \# indica haver diferença significativa do pré para o pós-treinamento no mesmo grupo.

Fonte: Elaborada pelos autores. 
CN tenha melhorado o quadro de composição corporal, estas mudanças foram semelhantes as do grupo CL.

Os principais achados neste trabalho foram: (1) melhora efetiva no somatório de dobras cutâneas e membros superiores para ambos os grupos; (2) a massa corporal e a massa magra não se modificaram após o período de treinamento de CN e CL. Coletivamente estes achados confirmam uma adaptação positiva nos valores relacionados à quantidade de gordura corporal, ao mesmo tempo em que não indicaram reduções de massa corporal durante o período. Tendo em consideração que a massa corporal é reduzida nessas pessoas ${ }^{13,14}$, é possível afirmar que, em um programa de curta duração (seis semanas) de caminhada, podem-se obter ganhos de composição corporal sem o risco de redução de massa corporal. Estes achados também confirmam os encontrados em investigações anteriores as quais demonstram que a $\mathrm{CN}$ é uma ferramenta útil no combate à DP devido aos resultados positivos na funcionalidade e na mobilidade $^{22}$, bem como em sintomas depressivos e qualidade de vida. Além disso, a CN mostrou resultados melhores do que a CL na mobilidade funcional e autonomia ${ }^{15}$. Em virtude dessas adaptações conhecidas, esperava-se que o perfil de composição corporal fosse mais modificado na $\mathrm{CN}$, o que não foi confirmado.

Na composição corporal do indivíduo com DP, a variável massa magra pode apresentar atrofia por desuso ou descondicionamento físico, em decorrência das manifestações da doença ${ }^{12}$. Tal fato acaba por reduzir o potencial muscular, ocasionando movimentos descoordenados, quedas e baixa adesão aos exercícios físicos ${ }^{14}$.

Em um estudo em que se comparou a composição corporal de idosos saudáveis com sujeitos com DP, a variável de massa magra apresentou valores menores em relação aos indivíduos saudáveis. Tais resultados foram atribuídos à atrofia por desuso ou descondicionamento físico ${ }^{23}$. O próprio processo de envelhecimento já causa reduções de fibras musculares do tipo IIA, predis- pondo à fraqueza e fadiga muscular, em indivíduos com DP essa redução é mais significativa ${ }^{24}$.

Por sua vez, na pesquisa de Yilmaz et al. ${ }^{25}$, com 73 portadores da DP, observa-se uma importante redução de massa magra em relação ao grupo controle de 71 indivíduos. A massa corporal total foi semelhante, indicando que a sarcopenia, devido ao envelhecimento, pode ser mais intensa em pessoas com DP. De modo interessante, o estudo aqui apresentado mostra que o exercício físico aeróbico pode ser uma ferramenta útil a fim de diminuir estes prejuízos.

Em relação aos resultados das correlações entre o estadiamento da DP pela escala H\&Y e o IMC, bem como a escala H\&Y, com o \% da gordura corporal, não foi encontrada nenhuma correlação significativa nos dados da amostra, tanto no pré- quanto no pós-treinamento para ambos os grupos. Provavelmente, esses achados podem ser explicados pelo baixo número de sujeitos com estadiamento mais avançados na escala H\&Y em ambos os grupos de treinamento.

Entretanto, os sintomas principais da DP, como rigidez, bradicinesia e tremor, e o fenômeno do Freezing, podem acarretar limitação funcional do indivíduo. Com a progressão da doença, surgem alterações na postura e na marcha, o que contribui mais intensamente para o afastamento da prática de atividades físicas ${ }^{8}$. Além disso, o risco de quedas também prejudica o desempenho nos exercícios físicos. Em estágios leves, o risco de queda do indivíduo com DP equipara-se ao de queda de idosos saudáveis. No estágio avançado, no entanto, o aumento do risco de quedas está relacionado às alterações cognitivas ${ }^{26,27}$.

Desta forma, o uso dos bastões de CN proporciona apoio e equilíbrio, gerando segurança para a prática da caminhada ${ }^{20}$. Assim, acreditase que a $\mathrm{CN}$ pode ser um modo de se exercitar tão efetivo quanto o da CL para a população com DP, pois, além de promover benefícios à saúde em geral, possibilita menor impacto nas articulações dos membros inferiores com o uso dos bastões e pode ser uma forma segura de realizar atividade física devido à maior estabilida- 
de de tronco ${ }^{28}$, tornando viável que indivíduos portadores de DP a pratiquem sozinhos ${ }^{29}$.

Além disso, a CN gera maior gasto energético por exigir mais trabalho mecânico ${ }^{12}$ e maior produção de ativação muscular dos membros superiores e abdominais, o que pode justificar a mudança nos valores de circunferências de MMSS $^{30}$ encontrados nos resultados do atual estudo. Estas alterações biomecânicas e neuromusculares da $\mathrm{CN}$ aliadas ao maior perímetro de MMSS indicam que são possíveis adaptações de força muscular com a prática da $\mathrm{CN}$. Adicionalmente, observou-se que houve uma maior redução $(5,5 \%)$ do somatório das dobras cutâneas referentes aos membros superiores no grupo $\mathrm{CN}$, em comparação ao grupo CL.

No entanto, o protocolo de treinamento proposto nesta pesquisa, tanto para $\mathrm{CN}$ quanto para $C L$, não foi eficaz na promoção de mudanças significativas na massa corporal, no IMC, nos MMII, nas massas magra, gorda e óssea, bem como nos percentuais de gordura, de massa muscular e de massa óssea da amostra. Esse resultado é diferente do encontrado em um recente estudo de Barbalho et al. ${ }^{26}$, em que se avaliaram os efeitos de 12 semanas de treinamento de resistência (TR) muscular sobre a força, capacidade funcional e composição corporal de 15 sujeitos com DP. Os autores concluíram que o TR realizado de forma isolada foi capaz de gerar positivas mudanças na composição corporal e ganhos na força e na funcionalidade de pessoas com DP. Os resultados, no atual estudo, podem ser explicados pelo curto período de treinamento e que investigações com um período de treinamento de CN e CL superior a seis semanas podem indicar modificações específicas na composição corporal entre modelos de treinamento de caminhada com e sem bastões em pessoas com DP.

Este estudo tem limitações que necessitam ser discutidas. Os resultados aqui apresentados devem ser limitados para intervenções de até seis semanas. Embora os achados atuais sejam interessantes, pela relativa curta duração, estudos com maior duração são necessários para verificar as adaptações de composição corporal em pessoas com DP. Além disso, esta pesquisa não apresenta grupo controle, portanto os achados devem ser analisados com cuidado. É importante notar que, devido à característica neurodegenerativa do distúrbio analisado, as diferenças encontradas poderiam ser intensificadas com a adição do grupo controle. Outra limitação é não ter sido avaliado o gasto energético dos sujeitos durante as sessões de treinamento, bem como o nível de atividade física diário. Tais informações poderiam ser agregadas aos resultados, por esse motivo, sugere-se que novas investigações sejam realizadas.

\section{Conclusõo}

O programa de treinamento de $\mathrm{CN}$ e $\mathrm{CL}$ promoveu melhora efetiva para ambos os grupos nos parâmetros de composição corporal, tais como relação cintura/quadril, somatório de dobras cutâneas de membros superiores e inferiores e no somatório total. Assim, este estudo indica que a CN é tão eficaz quanto a CL para a composição corporal de pessoas com DP.

\section{Agradecimentos}

Os autores expressam seus agradecimentos pelos financiamentos recebidos ao Laboratório de Pesquisa do Exercício (LAPEX) (n. 028/2015), à Coordenação de Aperfeiçoamento de Pessoal de Nível Superior (CAPES) (n.1159844), ao Conselho Nacional de Desenvolvimento Científico e Tecnológico (CNPq) (n. 483510/2013-0), FIPEHCPA $\left(n^{\circ} 140051\right)$ e à Decathlon Stores - NewFeel em nome do senhor Érik Pflieger. São igualmente gratos ao Grupo de Pesquisa Locomotion da Universidade Federal do Rio Grande do Sul e ao Grupo de Pesquisa em Análises de Movimento e Neurorreabilitação da Universidade Federal de Ciências da Saúde de Porto Alegre pelas discussões, comentários e apoio intelectual para este estudo. E expressam seus sinceros agradecimentos à Associação de Parkinson do Rio Grande do Sul (APARS) e a todos os voluntários do estudo. 


\section{Financiamento}

Fundo de Incentivo a Pesquisas (FIPEHCPA) $N^{\circ}$ 140051, LAPEX (n. 028/2015), CAPES (n.1159844), CNPq (n. 483510/2013-0), Decathlon Stores (Bastões - Newfeel Oficial).

\section{Referências}

1. Rafferty MR, Schmidt PN, Luo ST, Li K, Marras C, Davis TL, et al. Regular exercise, quality of life, and mobility in Parkinson's disease: a longitudinal analysis of national Parkinson foundation quality improvement initiative data. J Parkinson Dis. 2017;7(1):193-202.

2. Dos Santos EM, Ziegler JR, Ferreira FV. Doença de Parkinson: revisão bibliográfica. Disciplinarum Scientia| Saúde. 2016;8(1):115-29.

3. Monteiro EP, Franzoni LT, Cubillos DM, Fagundes AO, Carvalho AR, Oliveira HB, et al. Effects of Nordic walking training on functional parameters in Parkinson's disease: a randomized controlled clinical trial. Scand J Med Sci Sports. 2017;27(3):351-8.

4. Franzoni LT, Monteiro EP, Oliveira HB, da Rosa RG, Costa RR, Rieder C, et al. Week Nordic and free walking improve postural balance in Parkinson's disease. Sports Med Int Open. 2018;2(01):E28-E34.

5. Cugusi L, Solla P, Serpe R, Carzedda T, Piras L, Oggianu M, et al. Effects of a Nordic walking program on motor and non-motor symptoms, functional performance and body composition in patients with Parkinson's disease. NeuroRehabilitation. 2015;37(2):245-54.

6. Bang DH, Shin WS. Effects of an intensive Nordic walking intervention on the balance function and walking ability of individuals with Parkinson's disease: a randomized controlled pilot trial. Aging Clin Exp Res. 2017;29(5):993-9.

7. Delabary M, Komeroski IG, Monteiro EP, Costa RR, Haas NA. Effects of dance practice on functional mobility, motor symptoms and quality of life in people with Parkinson's disease: a systematic review with meta-analysis. Aging Clin Exp Res. 2017:1-9.

8. Araújo LP, Rodrigues APG. Exercício físico e avaliação da capacidade funcional de um idoso com Parkinson. Motricidade. 2018;14(SI).
9. Kang TW, Lee JH, Cynn HS. Six-week Nordic treadmill training compared with treadmill training on balance, gait, and activities of daily living for stroke patients: a randomized controlled trial. J Stroke Cerebrovasc Dis. 2016;25(4):848-56.

10. Peyré-Tartaruga LA, Monteiro EP. Perspective: A new integrative approach to evaluate pathological gait: locomotor rehabilitation index.2016;1(2):86-90

11. Peyré-Tartaruga LA, Coertjens M. Locomotion as a powerful model to study integrative physiology: efficiency, economy, and power relationship. Front Physiol. 2018;9:1789.

12. Pellegrini B, Peyré-Tartaruga LA, Zoppirolli C, Bortolan L, Savoldelli A, Minetti AE, Schena F. Mechanical energy patterns in nordic walking: comparisons with conventional walking. Gait Posture. 2017;(51):234-8.

13. Gomeñuka NA, Oliveira HB, Silva ES, Costa RR, Kanitz AC, Liedtke GV, et al. Effects of Nordic walking training on quality of life, balance and functional mobility in elderly: a randomized clinical trial. PloS One. 2019;14(1):e0211472.

14. Kim S, Kim Y, Park, SM. Body mass index and decline of cognitive function. PloS One. 2016;11(2):e0148908.

15. Arcila DMC, Monteiro EP, Gomeñuka NA, PeyréTartaruga LA. Metodologia e didática pedagógica aplicada ao ensino da caminhada nórdica e livre para pessoas com doença de Parkinson. Cadernos de formação RBCE. 2018;8(2).

16. Aiello M, Eleopra R, Rumiati RI. Body weight and food intake in Parkinson's disease. A review of the association to non-motor symptoms. Appetite. 2015;84:204-11.

17. Hughes AJ, Daniel SE, Kilford L, Lees AJ. Accuracy of clinical diagnosis of idiopathic Parkinson's disease: a clinico-pathological study of 100 cases. J Neurol Neurosurg Psychiatry. 1992;55(3):181-4.

18. Marfell-Jones $\mathrm{M}$, et al. International standards for anthropometric assessment. Potchefstroom, South Africa: ISAK; 2006

19. Siri WE. Body composition from fluid spaces and density: analysis of methods. 1961 Nutrition. 1993;9(5):480-91.

20. Sofuwa O, Nieuwboer A, Desloovere K, Willems $\mathrm{AM}$, Chavret F, Jonkers I. Quantitative gait analysis in Parkinson's disease: comparison with a healthy control group. Arch Phys Med Rehabil. 2005;86(5):1007-13. 
21. Scalzo P, Kümmer A, Cardoso F, Teixeira AL. Increased serum levels of soluble tumor necrosis factor- $\alpha$ receptor-1 in patients with Parkinson's disease. J Neuroimmunol. 2009;216(1):122-5.

22. Monteiro EP, Wild LB, Martinez FG, Souza, AP, Peyré-Tartaruga, LA. Aspectos biomecânicos da locomoção de pessoas com doença de Parkinson: revisão narrativa. Rev Bras Ciênc Esporte. 2017;39(4):450-7.

23. Bonjorni LA, Jamami M, Di Lorenzo VAP, Pessoa BV. Influência da doença de Parkinson em capacidade física, função pulmonar e índice de massa magra corporal. Fisioter Mov. 2017;25(4).

24. Bernhardt D, Müller HP, Ludolph AC, Dupuis L, Kassubek J. Body fat distribution in Parkinson's disease: An MRI-based body fat quantification study. Parkinsonism Relat Dis. 2016;33:84-9.

25. Yilmaz O, Donmez B, Inci I, Duran E, Cakmur R. Evaluation of sarcopenia in Parkinson's disease. Movement Disorders. 2015;30(1):4162.

26. Barbalho M, Monteiro EP, Costa RR, Raiol R. Effects of low-volume resistance training on muscle strength and functionality of people with Parkinson's disease. (In Press).
27. Wild LB, Lima DB, Balardin JB, Rizzi L, Giacobbo $\mathrm{BL}$, Oliveira HB, et al. Characterization of cognitive and motor performance during dual-tasking in healthy older adults and patients with Parkinson's disease. J Neurol. 2013;260(2):580-9.

28. Gougeon MA, Zhou L, Nantel J. Nordic walking improves trunk stability and gait spatial-temporal characteristics in people with Parkinson disease. NeuroRehabilitation. 2017:1-6.

29. Pellegrini B, Peyré-Tartaruga LA, Zoppirolli C, Bortolan L, Bacchi E, Figard-Fabre H, Schena F. Exploring muscle activation during Nordic walking: a comparison between conventional and uphill walking. PloS One. 2015;10(9):e0138906.

30. Cugusi L, Manca A, Yeo TJ, Bassareo PP, Mercuro G, Kaski JC. Nordic walking for individuals with cardiovascular disease: a systematic review and meta-analysis of randomized controlled trials. Eur J Prev Cardiol. 2017;24(18):1938-55. 